\title{
Brain Tumor Detection
}

\author{
Mr. Akash Poduval ${ }^{1}$, Mr. Akshay Saste ${ }^{2}$, Ms. Rajeshwari Bathe ${ }^{3}$, Mr. Shubham Sethiya ${ }^{4}$, \\ Prof. Pavan Kulkarni ${ }^{5}$ \\ Student, Comp Dept, KJEI's Trinity College of Engineering and Research Pisoli, Pune, India ${ }^{1,2,3,4}$ \\ Guide, Comp Dept, KJEI's Trinity College of Engineering and Research Pisoli, Pune, India ${ }^{5}$
}

\begin{abstract}
Brain cancer develops because of unusual cell growth within the brain. Brain Cancer generally classified into two types benign and malignant tumours. Malignant Cancer are fast growing cancerous tissues. Benign are slow growing, stagnant cancerous Cancer. Most of the cancer are life threatening, brain tumour being one among them. Primary brain cancer originates in the brain. In the Secondary type of brain cancer the tumor expansion into the brain results from other parts of the body. Imaging brain cancer with more accuracy plays pivotal role in the diagnosis of tumours. It involves high resolution techniques like MRI , CT ,PET etc. MRI is a important mean for studying the body's visceral structures. MRI is widely used because it gives better quality images of the brain and cancerous tissues compared with the other medical imaging techniques such as X-Ray or Computed Tomography (CT). In this proposed system we are creating python application for detection of brain cancer base on three step.1. Classification of MRI image to determining the brain cancer base on type.2.Prevention that should be taken to overcome this deadly disease.3.Care that should be taken by normal people to save themselves from brain cancer.
\end{abstract}

Keywords: Brain cancer, CNN ,OPENCV, Prevention, Classification, Care

\section{INTRODUCTION}

Brain tumor detection and segmentation is one of the most challenging and time consuming task in medical image processing. MRI (Magnetic Resonance Imaging) is a medical technique, mainly used by the radiologist for visualization of internal structure of the human body without any surgery. MRI provides plentiful information about the human soft tissue, which helps in the diagnosis of brain tumour. Accurate segmentation of MRI image is important for the diagnosis of brain tumor by computer aided clinical tool. After appropriate segmentation of brain MR images, tumor is classified to malignant and benign, which is a difficult task due to complexity and variation in tumor tissue characteristics like its shape, size, gray level intensities and location. Taking in to account the aforesaid challenges, this research is focussed towards highlighting the strength and limitations of earlier proposed classification techniques discussed in the contemporary literature. Besides summarizing the literature, the paper also provides a critical evaluation of the surveyed literature which reveals new facets of research.

\section{LITERATURE SURVEY}

\section{A Survey on Brain Tumor Detection Using Image Processing Techniques" Luxit Kapoor, Sanjeev Thakur IEEE 2017[1]}

The primary goal of medical imaging is to extract meaningful and accurate information from these images with the least error possible. Out of the various types of medical imaging processes available to us, MRI is the most reliable and safe. It does not involve exposing the body to any sorts of harmful radiation. This MRI can then be processed, and the tumor can be segmented. The whole process of detecting brain tumor from an MRI can be classified into four different categories: Pre- Processing, Segmentation, Optimization and Feature Extraction

Detection of Brain Tumor from MRI images by using Segmentation \&SVM" World Conference on Futuristic Trends in Research and Innovation for Social Welfare 2016[2]

In this paper we propose adaptive brain tumor detection, Image processing is used in the medical tools for detection of tumor, only MRI images are not able to identify the tumorous region in this paper we are using K-Means segmentation with preprocessing of image. Which contains denoising by Median filter and skull masking is used. Also we are using object labeling for more detailed information of tumor region. 
"Detection of Tumor in MRI Images Using Artificial Neural Networks" Aqhsa Q. Syed, K. NarayananInternational Journal of Engineering Research \& Technology (IJERT) September 2014[3]

Automatic defects detection in MR images is very important in many diagnostic and therapeutic applications. This work has introduced one automatic brain tumor detection method to increase the accuracy and yield and decrease the diagnosis time. The goal is classifying the tissues to two classes of normal and abnormal. MR images that have been used here are MR images from normal and abnormal brain tissues. This method uses from neural network to do this classification. The purpose of this project is to classify the brain tissues to normal and abnormal classes automatically, that saves the radiologist time, increases accuracy and yield of diagnosis.

\section{PROPOSED SYSTEM}

In a proposed system we are making python application.

This system provides gist of technologies which can be used to predict brain cancer and the result will be classification of brain MRI to detect the image depicts brain cancer and provide preventation and care that should be taken for brain cancer.

In that the work is divided into three phase

1. Classification to classify the brain MRI images

2. Prevention that should be taken to save from this type of deadly disease.

3. Care that should be taken to person suffering from brain cancer.

\section{SYSTEM ARCHITECTURE}

Following diagram is our system's architecture diagram:

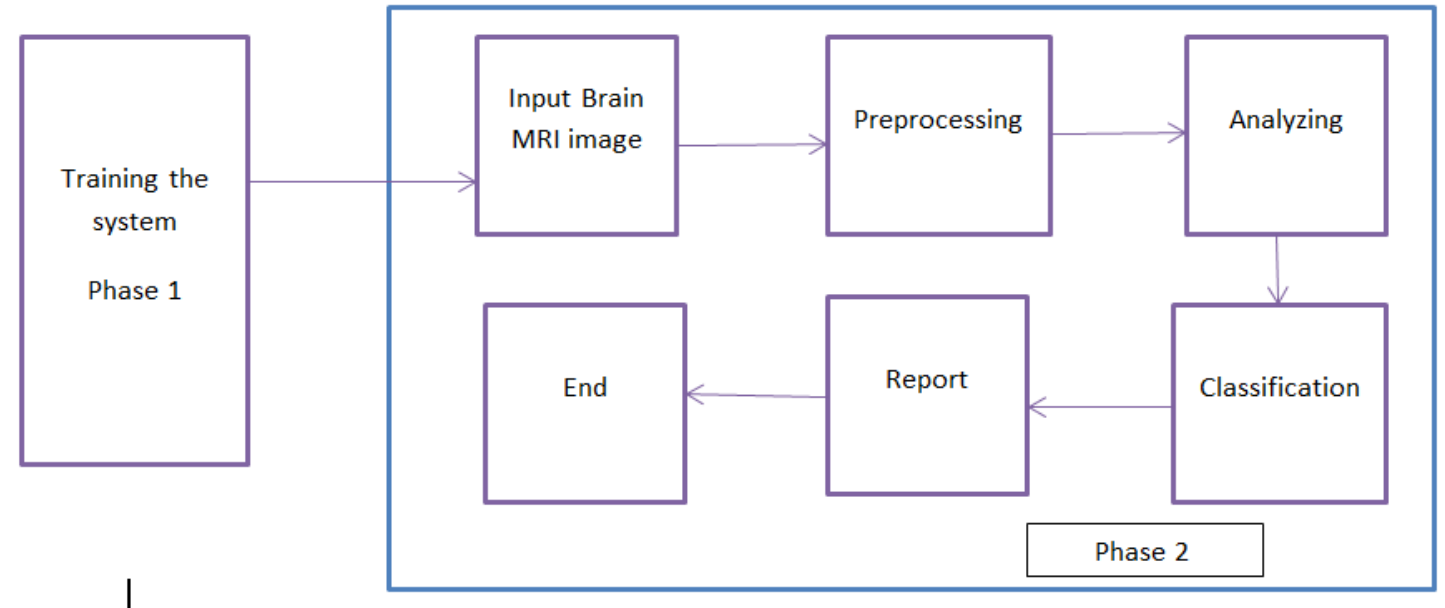

Figure 1: system architecture

In this proposed system 3 main modules are use they are:-

1. Dataset training

2. Pre-processing

3. Analysing

4. Classification

Training Data :- In training dataset module we take Brain MRI images form user and trained them for the further stage.

Pre-processing :- In pre-processing module the data which is trained in last stage is given for the further processing and move forward to the analysing stage .

Analysing :- In this analysing stage data that is pre -processed is analysed step by step and determine and move forward for classification 


\section{International Journal of Advanced Research in Computer and Communication Engineering}

Vol. 8, Issue 3, March 2019

Classification:- In Classification module the MRI images is input into system to get classify into different types of brain cancer.

\section{METHODOLOGIES}

1. CNN algorithm Network: In that MRI images is process and analyse and classify So using CNN algorithm.

Convolution Neural: In machine learning, a convolutional neural network is a class of deep, feed-forward artificial neural networks that has successfully been applied to analysing visual imagery. Convolution neural network algorithm is a multilayer perceptron that is the special design for identification of two-dimensional image information. Always has more layers: input layer, convolution layer, sample layer and output layer. CNNs use relatively little pre-processing compared to other image classification algorithms. This means that the network learns the filters that in traditional algorithms were hand-engineered.

2. Open Cv: OpenCV (Open Source Computer Vision Library) is an open source computer vision and machine learning software library. OpenCV was built to provide a common infrastructure for computer vision applications and to accelerate the use of machine perception in the commercial products. In this system open cv library is used for image processing for the purpose of classification of MRI images.

\section{CONCLUSION}

The proposed system can efficiently detect brain cancer and can also find the boundary extraction of cancer. Size and stage of tumour is described. MRI images are best suitable for brain detection. In this system Digital Image Processing Techniques are used for brain cancer detection by MRI images. Prevention is provided and care is provided for person suffering from brain cancer to overcome it .

\section{FUTURE WORK}

The future scope of this project can be to work on algorithms for colour images as well as identification of the type of the brain cancer.

\section{RESUlT}

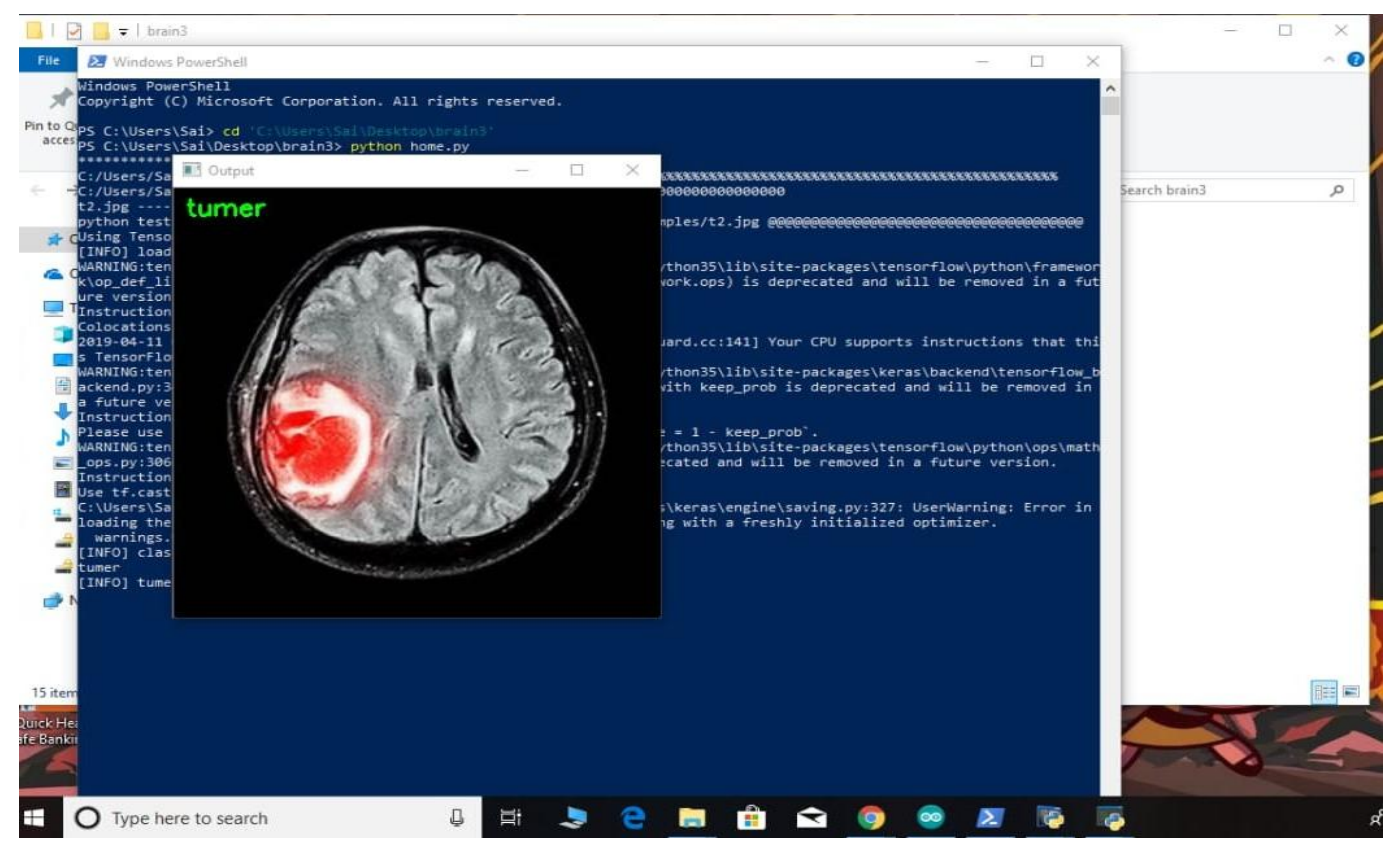




\section{International Journal of Advanced Research in Computer and Communication Engineering}

Vol. 8, Issue 3, March 2019

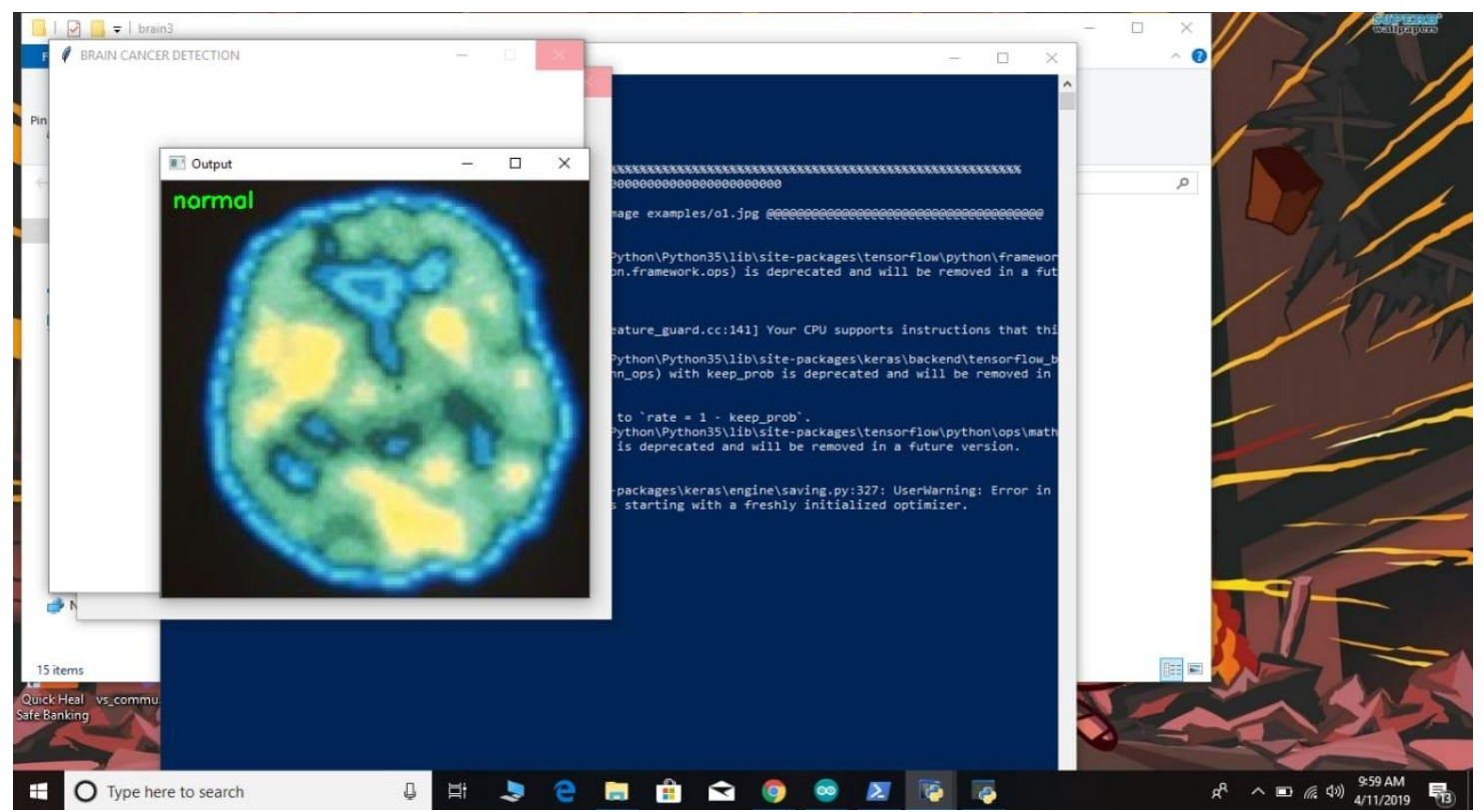

\section{REFERRENCES}

[1]. A Survey on Brain Tumor Detection Using Image Processing Techniques" Luxit Kapoor, Sanjeev Thakur IEEE 2017.

[2]. Detection of Brain Tumor from MRI images by using Segmentation \&SVM" World Conference on Futuristic Trends in Research and Innovation for Social Welfare 2016

[3]. "Detection of Tumor in MRI Images Using Artificial Neural Networks" Aqhsa Q. Syed, K. Narayanan- International Journal of Engineering Research \& Technology (IJERT) September 2014.

[4]. "Classification of Brain Cancer Detection by using Magnetic Resonance Imaging" Vishal S. Shirsat, Seema S. Kawathekar--International Journal of Engineering Research \& Technology (IJERT) Vol. 3 Issue 2, February - 2014

[5]. E.Lashkari, "A Neural Network- Based Method for Brain Abnormality Detection in MR Images Using Zernike Moments and Geometric Moments", International Journal of Computer Applications, Vol.

[6]. http://www.radiologyinfo.org/

[7]. http://www.webmd.com/brain/magneticresonance- imaging-mri-of-the-head

[8]. https://blogs.nvidia.com/blog/2016/07/29/ whats-difference-artificialintelligencemachinelearning-deep-learning-ai 